\title{
Verhaltenstherapie - Panta Rhei? 15 Jahre Psychotherapeutengesetz - and Where Are We?
}

\author{
Iver Hand \\ Medizinische Fakultät der Universität Hamburg, Verhaltenstherapie Falkenried, Hamburg, Deutschland
}

«Alles fließt und nichts bleibt; es gibt nur ewiges Werden und Wandeln» (Platon, frei nach Heraklit); «Einheit in der Vielheit und Vielheit in der Einheit» (zit. nach Panta rhei. Wikipedia) - ist das Trost oder Enttäuschung für einen Verhaltenstherapeuten der ersten Stunde? Können wir noch definieren, was Verhaltenstherapie (VT) heute beinhaltet, i.e. «where it is»? (frei nach Kanfer: «Join the client where he is»). Und: «Is it, where it says to be?» [Hand, 2008]. Kanfer forderte: «Think flexible» - ist daraus inzwischen ein «too flexible» geworden?

13 Jahre sind nun seit dem Editorial zur Kernfrage «Verhaltenstherapie - Quo vadis seit dem PTG?» [Hand, 2001] vergangen. 2 Jahre später drängte sich die Frage auf: «Verhaltenstherapie - Schutz vor Mythologie und Magie in der Psychotherapie?» [Hand, 2003]. Und heute nun die Frage: Wo bist $\mathrm{Du}$ angekommen, VT? Ist dein Label noch ein Markenzeichen für definierte Inhalte einer Krankenbehandlung oder eher ein Sammelbegriff für etliche heterogene psychotherapeutische, psychologische oder (pseudo-)philosophische/ religiöse Zeitströmungen mit dem Ziel einer Verhaltensmodifikation zur Erhöhung «psychischer Wellness»? Oder anders gefragt: Ist noch VT drin, wo VT drauf steht?

Diese Frage wird zum Teil kontrovers diskutiert - nicht zuletzt im und um das Gutachterverfahren in der RichtlinienVT. Die Gratwanderung zwischen rigiden Normvorgaben und kreativem Experimentieren ist ebenso notwendig wie schwierig. Das zeigt sich auch am Begriff der evidenzbasierten Techniken und Verfahren - er wird gebraucht zur Qualitätssicherung und missbraucht zur Entwicklungsverhinderung. Die Kriterien für einen Evidenznachweis sind uneinheitlich, besonders für die sogenannte störungsspezifische Psycho- und Verhaltenstherapie, die es so gar nicht geben kann [Linden, 2013].

Was haben die «zweite» und die «dritte Welle» der VT bewirkt? Von der Nordsee wissen wir, dass Wellen das Schwimmen erst richtig schön machen können. Werden daraus aber Sturmfluten, dann können sie großen Schaden anrichten. War die «kognitive Wende» (2. Welle) eine Sturmflut für die zurzeit noch junge VT? Für viele «klassische» Verhaltenstherapeuten ist sie dies bis heute, obwohl kognitive Verhaltenstherapie (KVT) international längst der am häufigsten genutzte Sammelbegriff für VT ist. Dabei hat die dritte Welle - mit der neoanalytisch beeinflussten Wiederentdeckung prägender Emotionen und früher biografischer Erfahrungen - längst andere Schwerpunkte in die VT eingeführt, ohne dass eine Umbenennung etwa in «emotionale Verhaltenstherapie» erfolgte. Allerdings wurden bis 2008 über ein Dutzend Vorschläge für eine Umbenennung von VT bzw. KVT publiziert [Hand, 2006; 2008]. Einige Kollateralschäden dieser Wellen oder Fluten auf längst bewährte VT-Vorgehensweisen werden im Folgenden angeführt.

\section{Die kassenfinanzierte «Richtlinien-Psychotherapie» heute}

Zur kassenfinanzierten Psychotherapie (PT)-Versorgung gehören heute neben der Richtlinien-PT auch die Therapien im «Erstattungsverfahren», im «Hausarztmodell» und in der «integrierten Versorgung», die jeweils von einigen Kassen in einigen Bundesländern angeboten werden. Nur über erstere liegen mir konkrete Zahlen vor:

Mehr als 50\% aller Richtlinien-VT sind Kurzzeittherapien (KZT), zwei Drittel werden ohne Gutachterverfahren bewilligt [Techniker Krankenkasse (TK), 2013].

Von den Versicherten der TK nehmen 50\% nur bis zu maximal 12 Therapiestunden, weitere 34\% 13-25 und nur 16\% mehr als 25 in Anspruch; lediglich 1\% erhält eine Gruppentherapie, und nur 10\% vollziehen einen Therapeutenwechsel [TK, 2013; Malinke, 2014].

Liegt darin ein Qualitätsnachweis (gute Therapeuten mit guten Therapien brauchen wenige Therapiesitzungen, um erfolgreich zu sein) oder eher ein Indikator für Qualitätsmängel (schlechte Therapeuten meiden das Gutachterverfahren; sie suchen sich meist nur die «leichten» Fälle; sie beenden Thera-

\section{KARGER \\ Fax +497614520714 \\ Information@Karger.com}

www.karger.com (c) 2014 S. Karger GmbH, Freiburg

1016-6262/14/0243-0151\$39.50/0

Accessible online at:

www.karger.com/ver
Prof. Dr. med. Iver Hand

Falkenried 7, Postfach 201861

20208 Hamburg, Deutschland

hand@vt-falkenried.de 
pien vor erfolgreichem Abschluss, wenn eigentlich ein Verlängerungsantrag in das Gutachterverfahren zu geben wäre)? Dazu scheint es keine Daten zu geben.

Weitere Fragen liegen nahe: Gehen die meisten Therapeuten und Patienten vielleicht nur sehr sorgsam und zurückhaltend mit den großzügig angebotenen Psychotherapieleistungen um? Ist Psychotherapie für Patienten längst nicht so attraktiv wie wir gerne glauben? Wird die Indikation für eine PT viel zu häufig gestellt (siehe dazu ein Modellprojekt in Bad Oeynhausen: Nur in ca. 50\% der Fälle wurde im Erstkontakt eine PT angeboten; den übrigen Betroffenen wurden alternative Hilfsangebote nahegelegt; [Buchmann und Wörder, 2013])? Oder wird zu vielen Patienten aufgrund der strukturellen Gegebenheiten (siehe unten) die für sie «falsche» Psychotherapieform angeboten? Bezüglich dieser Frage drängen sich Parallelen zur überraschend geringen Inanspruchnahme bzw. hohen Abbruchquote von Suchtberatungen durch pathologische Glücksspieler auf [Hand und Kegat, 2013].

Im Gutachterverfahren wird eine andere VT-Welt wahrgenommen: Ein Trend zu immer mehr Therapiesitzungen! Zum einen geht dies vermutlich auf eine andere Klientel als in den KZT zurück. Zum anderen könnten aber auch Therapeutenvariablen und kassenspezifische Klientel einen Einfluss haben. Auffallend ist auch die erhebliche Ungleichverteilung der Durchführung der 3 kassenzugelassenen Psychotherapieformen in den Bundesländern [TK, 2013]: In Bremen und Hamburg werden mit 16 bzw. 24\% vergleichsweise wenige VT durchgeführt; demgegenüber stehen auffallend viele analytische PT $($ Bremen $=35 \%$; bundesweiter relativer Anteil: tiefenpsychologisch fundierte PT 43\%, Psychoanalyse 21\% und VT $36 \%$ ). Hier liegt eine Versorgungsschieflage vor, die vor allem historisch-systembedingt ist und etliche Patienten möglicherweise massiv benachteiligt.

Wir wissen nicht, wie viele Kassen diesen Mangel und lange Wartezeiten über das Erstattungsverfahren auszugleichen versuchen. Wir wissen aber, dass etliche Nachwuchstherapeuten mit qualifiziertem Abschluss, aber ohne Kassensitz, davon recht gut leben können.

\section{Das Gutachterverfahren in der Richtlinien-VT}

Das Gutachterverfahren ist seit Langem umstritten. In der Praxis läuft es zwar meist recht problemlos, etliche Therapeuten fühlen sich aber auch unzulässig supervidiert bzw. bevormundet und empfinden die Regeln bezüglich neuer Therapieentwicklungen als zu restriktiv und das Procedere als viel zu bürokratisiert. Einige Kassen (z.B. TK), meinen, dass das Verfahren «keine Steuerungsfunktion» habe und deshalb abgeschafft werden sollte. Dagegen möchte der GKV-Spitzenverband (GKV-SV) das Verfahren lediglich reformieren, unter anderem mit nur 2 Begutachtungen, jeweils nach 25 und nach 50 Sitzungen [Malinke, 2014].

Die Fakten für die VT: 2009 wurden ca. 1 Million Richtlinien-PT durchgeführt, begleitet von etwa 310000 (Ober-)Gutachten. Die Kosten für die Therapien lagen bei 1,2-1,5 Milli- arden EUR, etwa 10\% des Gesamtbudgets für die ambulante medizinische Versorgung der Bevölkerung. Für das (Ober-) Gutachterverfahren wurden 28-30 Millionen EUR aufgewendet [Malinke, 2014]. 2013 wurden ca. 172000 VT-Gutachten erstellt; Nichtbefürwortungen erfolgten bei 3,2\%, und bei weiteren 7,5\% wurden Änderungen veranlasst. Es wurden aber nur 1029 Obergutachten beantragt - also lediglich bei ca. $20 \%$ der ca. 5200 Nichtbefürwortungen! Davon wurden 19\% nicht befürwortet, und bei $25 \%$ wurden Änderungen veranlasst [KBV, 2013]. Da die meisten Therapeuten zu den Kritikpunkten der Erstgutachter inhaltlich Stellung beziehen, stellt das Obergutachterverfahren also keineswegs die Ergebnisse des Gutachterverfahrens infrage! Es bietet vielmehr die Möglichkeit, Irrtums- bzw. Fehlerkorrekturen auf beiden Seiten vorzunehmen.

Aus Sicht einiger Kassen werden viel zu wenige Anträge «abgelehnt» - ist das aber wirklich ein Kriterium für «schlechte» Arbeit der Gutachter? Viel entscheidender dürfte sein, dass ein guter Teil der (Ober-)Gutachter einen kollegialen Austausch mit den Therapeuten pflegt, was in den Statistiken nur rudimentär auftaucht.

Der Verband der Ersatzkassen [VdEK, 2013] diskutiert die Einrichtung regionaler «intelligenter Koordinierungsstellen» (iKS) als Ersatz für das Gutachterverfahren. In den iKS sollten «unabhängige» (Frage: Wer oder was ist das?) Psychotherapeuten mit den Patienten «gemeinsam und ohne Einflussnahme der Krankenkassen» klären, «welches Therapieverfahren nach evidenzbasierten Kriterien für die jeweilige Erkrankung besonders geeignet ist». Haben die Mitglieder des Thinktanks, die diese Idee entwickelt haben, jemals den Diskussionen zwischen der und innerhalb von Therapieschulen zu dieser Frage gelauscht - und sind sie trotzdem frohen Mutes?

Ein laufendes Experiment zur Einstellung des Gutachterverfahrens ist z.B. das Hausarztmodell der AOK und BKK in Baden-Württemberg [AOK, 2012], an dem 10000 Patienten und deren Ärzte und Psychotherapeuten teilnehmen. Damit sollen kurze Wartezeiten, eine enge Vernetzung aller Behandler eines Patienten und zügige Behandlungsabläufe erreicht werden. Dazu werden den Therapeuten finanzielle Anreize hinsichtlich rascher Terminvergaben und kurzer Therapiezeiten gegeben, deren Nutzen für die Patienten allerdings noch belegt werden muss. Neben den Richtlinien-Therapien sind auch (zumindest bei Alkoholsucht) systemische Therapie, Schematherapie und interpersonelle Therapie zugelassen.

Auch die Deutsche Gesellschaft für Psychiatrie und Psychotherapie, Psychosomatik und Nervenheilkunde [DGPPN, 2014] kritisiert die Richtlinien-PT, «die mit ihrem starren Gerüst den Bedürfnissen der psychisch erkrankten Menschen nicht gerecht werde»; sie müsste um «neue, nachweislich hochwirksame störungsorientierte Methoden und störungsübergreifende Techniken» erweitert werden. Die Umsetzung müsse von «kurzzeitig intensiven (Interventionen) (...) bis zu jahrelanger niederfrequenter psychotherapeutischer Unter- 
stützung reichen». Dabei solle den «Fachärzten für Psychiatrie und Psychotherapie eine zentrale und spezifische Rolle» zukommen.

Den inhaltlichen Forderungen werden die meisten psychologischen Psychotherapeuten vermutlich voll zustimmen, der vorgeschlagenen berufspolitischen Konsequenz wohl eher nicht.

Bei etwa 14000 psychologischen, aber nur etwa 5000 ärztlichen Psychotherapeuten/Psychosomatikern (jeweils ohne Kinder- und Judenglichenpsychotherapeuten) in 2010 und 2013 - und davon jeweils 47\% psychologischen und nur 13\% ärztlichen Verhaltenstherapeuten [Stoppe, 2013; KBV, 2013] - dürfte dieses Argument auch kaum durchsetzbar sein.

Im Bereich der Richtlinien-PT und des Gutachterverfahrens sind die Dinge also in heftigem Wellengang, der möglicherweise bessere Versorgungsmöglichkeiten für die Patienten hervorbringen wird, sich aber auch zu einer Sturmflut mit erheblichen Schäden entwickeln könnte. Als experimentierfreudige Verhaltenstherapeuten sehen wir den Ergebnissen mit Spannung entgegen.

\section{Die Rolle der Ausbildungsinstitute}

Die Akademisierung der VT, insbesondere seit Verabschiedung des Psychotherapeutengesetzes für Psychologen, beinhaltet oft auch eine Theoretisierung im negativen Sinne.

In der Medizin wurde früher ironisch gespottet: «Der Internist weiß alles, der Chirurg kann alles»; auf die Psychotherapie der 1970er und 1980er Jahre übertragen: «Der Analytiker weiß (alles), der Verhaltenstherapeut (be-)handelt (alles)». Als wir in London Anfang der 1970er Jahre erstmals Agoraphobiker mit Exposition in vivo in Kleingruppen in nur 3 ganztägigen Sitzungen innerhalb einer Woche erfolgreich behandelt hatten, hie $\beta$ es von analytischer Seite: «Aber das sind doch keine echten Agoraphobiker» - begründet alleine mit der Art und der Kürze der Therapie, die auf einem simpel wirkenden theoretischen Konzept basierte, aber daraus gar nicht abgeleitet war. Die Analytiker hatten eine viel komplexere Theorie. Diese Art des «Austauschs» ist weitgehend überwunden. In die tiefenpsychologisch fundierte Psychotherapie und in die VT ist inzwischen viel Gedankengut der jeweils anderen Schule eingegangen - zum Guten wie zum Nachteil für die gelehrte und praktizierte Therapie.

Die inzwischen überwiegend privatwirtschaftlich organisierten Ausbildungsinstitute für psychologische Psychotherapeuten sind inhaltlich an die Prüfungsordnung gebunden. Ein weiterer Einflussfaktor sind die durch Modetrends auf dem «Psycho-Markt» generierten Informationswünsche der Ausbildungskandidaten (die in diesem System auch die Kunden sind). In diesem Spannungsfeld müssen die Institute versuchen, ihre eigenen Vorstellungen umzusetzen. So sind die Ausbildungsgänge heute viel zu theorielastig geworden. Welche Rolle spielen dagegen die klassischen Ausbildungstugen- den der VT heute noch: Modeling durch den Supervisor, indem Ausbildungskandidaten (auch als Kotherapeuten) an dessen Therapien teilnehmen; Live-Supervision von Ausbildungstherapien durch den Supervisor (z.B. hinter der Einwegscheibe oder als Kotherapeut); In-vivo-(Expositions-) Therapien im Einzel-, Familien- und Gruppensetting - insbesondere bei Angst- und Zwangserkrankungen - mit dem Supervisor als Haupt- und später auch Kotherapeuten? Und wer vermittelt der «Google-Generation» des VT-Nachwuchses noch ein solides Studium der fachrelevanten Literatur der letzten Jahrzehnte?

Zumindest im Gutachterverfahren fällt unangenehm auf, dass viele Therapeuten offenbar keine übergeordnete VTStrategie beherrschen, aus der eine Hierarchisierung von Störungen, Symptomen und Problemen in der Form abgeleitet wird, dass auch ein individuell begründeter, hierarchisierter Behandlungsplan vorgelegt wird. Stattdessen werden die Patienten dann in etliche psychische Einzelbestandteile «psychoseziert», für die dann eine ganze Reihe unterschiedlicher Interventionen aufgezählt wird. Solche Behandlungspläne sind dann so richtig wie falsch [Hand, 2008]. Wo haben diese Therapeuten das gelernt?

Es darf auch nicht sein, dass akademische Therapeuten nach 4-5 Jahren universitären Studiums (z.B. der Psychologie) einen Großteil des Gelernten im VT-Institut gegen erhebliches Entgelt nochmals studieren müssen, um dann störungsspezifische Manuale zu lernen, anstatt eine klare Strategie der VT zu internalisieren, auf deren Grundlage dann störungsspezifische Einzelfall-Entscheidungen getroffen werden.

Es scheint auch, dass psychologische und ärztliche Ausbzw. Weiterbildungskandidaten kaum noch gemeinsam betreut werden, obwohl dies die Kleingruppenarbeit bei multimorbiden Patienten enorm bereichern würde [Hand et al., 2014]. Berufsgruppenspezifische Konkurrenzen und Arroganzen werden so zum Schaden der Patienten eher gefestigt als abgebaut.

\section{Tradition mit Evolution - nicht immer gelungen}

Eines der traditionellen «Filetstücke» der VT - die Expositionstherapie - hat unter den skizzierten Entwicklungen erheblichen Schaden genommen. Seit Langem wird bei Angstund Zwangsstörungen das Potential der Expositionstherapie gar nicht oder unzureichend genutzt. Die Hauptgründe scheinen wie folgt zu sein:

- Die Therapeuten haben keine oder eine schlechte Ausbildung erhalten (seine oben). Sie haben nicht erfahren, wie viel mit wenigen mehrstündigen Expositionssitzungen (Einzel oder Gruppe) innerhalb von 1-2 Wochen erreicht werden kann. Die entsprechenden Publikationen aus den 1970er und 1980er Jahren werden nicht mehr vermittelt. Stattdessen wird in bekannten Manualen z.B. zur Zwangsstörung 
von einer Therapeutenbegleitung eher abgeraten; bei Agoraphobie werden wöchentlich einmalige In-vivo-Sitzungen zur Bearbeitung von jeweils nur einer phobischen Situation propagiert [Hand, 2013]. Beides führt für viele motivierte Patienten $\mathrm{zu}$ unnötiger und möglicherweise demotivierender Therapieverlängerung.

- Das Konzept der Exposition wird nicht voll genutzt. So werden neuerdings Achtsamkeitsübungen als Zusatz zur Exposition mit Reaktionsverhinderung (ERV) empfohlen. Patienten sollten jedoch die seit Jahrzehnten bekannte und evaluierte Exposition mit Reaktionsmanagement (ERM) erhalten, bei der Achtsamkeitstraining bereits enthalten ist [Hand, 2011; 2013]. Bei Agoraphobie und sozialer Phobie ist deren Anwendung in vivo in Kleingruppen besonders effektiv. Die Therapeuten werden dafür aber in der Regel gar nicht ausgebildet.

Die organisatorischen Probleme bei der Umsetzung von Exposition in vivo in den Einzelpraxen sind in den Richtlinien gelöst. Gut begründete mehrstündige Sitzungen, selbst innerhalb einer Woche, werden problemlos akzeptiert. Trotzdem bestehen in der Praxis immer noch zahlreiche Probleme bei der Umsetzung [Bossert-Zaudig und Buxhoeveden, 2014]. Allerdings müssen die Kassen endlich die An- und Abfahrtszeiten der Therapeuten zum Übungsort adäquat honorieren [z.B. Hillebrand und Niedermeier, 2014]. Leider haben sich die meisten Therapeuten aber inzwischen an 50-min-Sitzungen, die einmal pro Woche oder gar seltener stattfinden, gewöhnt und ihre Sprechstunde entsprechend organisiert.

Störungsspezifische In-vivo-Gruppen sind in der Einzelpraxis organisatorisch noch wesentlich schwerer umsetzbar. Bereits heute wäre es aber möglich, an Orten mit Gruppenpraxen oder an Medizinischen Versorgungszentren (MVZ) für VT, Patienten aus Einzelpraxen nur zur Durchführung der massierten Gruppenexposition (als Ergänzung zur laufenden Einzeltherapie) zu überweisen. Entsprechende, sehr positive Erfahrungen haben wir wiederholt in der VT-Ambulanz des UKE gemacht [Hand et al., 2014]. Besonders bei den Angstund Zwangserkrankungen sollte die Richtlinien-VT mehr auf die Bedürfnisse der Patienten und weniger auf die der Therapeuten ausgerichtet werden.

Es ist noch ein langer Weg, bis wir in der VT der Angstund Zwangserkrankungen wieder das Input/Outcome-Ergebnis erreicht haben, das in den 1970er und 1980er Jahren entscheidend die Akzeptanz dieser Therapierichtung ermöglicht hat!

In anderen Störungsbereichen haben wir dagegen ein beeindruckendes Niveau erreicht, das in den ersten beiden Jahrzehnten der VT nicht erwartet werden konnte.

\section{Ausblick}

Was könnten/sollten die Kassen unternehmen, um unser deutsches «Leiden auf hohem Niveau» zu verringern? Die gesundheitspolitisch aufschlussreichste Maßnahme wäre, endlich die Kosten/Nutzen-Relation der zugelassenen und der aktuellen experimentellen Versorgungsmodelle dadurch zu prüfen, dass die Patientenkosten jeweils 2 Jahre vor und 2 Jahre nach einer VT/PT - aufgeschlüsselt nach Kurz- u. Langzeit-VT - bei den Patienten mit primär nichtorganischen und den primär organischen Erkrankungen dokumentiert werden. Eine zweite, einfachere wie dringliche Reform wäre die Sicherstellung psychotherapeutischer niederfrequenter VT/PT bei chronischen psychischen Erkrankungen (Gleichstellung in der Versorgung mit chronisch körperlich Kranken)!

Die Prävalenz psychischer Störungen hat in den vergangenen Jahren bzw. Jahrzehnten nicht zugenommen. Deren ambulante Behandlungskosten machen $10 \%$ des Kassenbudgets für ambulante medizinische Leistungen aus, aber 30\% der gesamtgesellschaftlichen Kosten (Arbeitsunfähigkeit; Frühverrentung bei psychischen Leiden mit 49, bei somatischen Erkrankungen mit 55 Jahren). Es gibt so viele qualifizierte Psychotherapeuten wie nie zu vor - aber scheinbar immer längere Wartezeiten für eine Psychotherapie, im weltweit mit Abstand besten psychotherapeutischen Versorgungssystem.

$\mathrm{Ob}$ und wie VT durch Veränderungen der Inhalte, der gesundheits-/kassenpolitischen Spielregeln und der Therapeutenausbildung zu einer Verbesserung der Situation beitragen kann, wird in diesem Beitrag andiskutiert. Nicht diskutiert wurde die Häufigkeit schwerer Konflikte einerseits am Arbeitsplatz und andererseits in engen Zweier- und Familienbeziehungen, die als (Mit-)Ursache für eine zu behandelnde Krankheitssymptomatik gelten (wann Indikation für Richtlinien-PT, wann für psychologische Lebensberatung?). Zu den Arbeitsplatzproblemen ist allerdings abschließend festzuhalten: Weder VT noch PT können offensichtliche gesellschaftliche Fehlentwicklungen - vom Turbokapitalismus des Investmentbankings, dessen Folgen uns alle bedrohen, bis zu den von Egoismus geprägten zunehmenden Top-down-Führungsstilen in Industrie und Behörden - neutralisieren. VT/PT können im besten Falle die Resilienz des Individuums verbessern - und vielleicht auch den Willen und die Fähigkeit zum Widerstand. 


\section{Literatur}

AOK-Hausarztmodell Südbaden, 2012. www.aok-gesundheitspartner.de/bw/arztundpraxis/arztnetze/pi lotprojekte/index_09186.html (Zugriff 14.08.2014).

Bossert-Zaudig S, von Buxhoeveden I: Therapieempfehlung versus Realität: Anwendung und Kosten leitliniengerechter Exposition in der ambulanten Verhaltenstherapie bei Zwangsstörungen. Ärztliche Psychotherapie und Psychosomatische Medizin 2014;2:8-11.

Buchmann M, Wörder M: Psychotherapeutische Erstversorgung in Bad Oeynhausen/Löhne. Psychotherapie Aktuell 2013;2:35-37.

Stoppe G: Es gibt keine Gesundheit ohne psychische Gesundheit. Dtsch Arztebl 2013;110;A543.

DGPPN: Durch Fachärzte erbrachte Psychotherapie stärken - Neustrukturierung der ambulanten Behandlung gefordert. Pressemitteilung, Nr. 15, 2014. http://www.dgppn.de/fileadmin/user_upload/_medien/download/pdf/pressemitteilungen/ 2014/2014-06-27_DGPPN-Pressemitteilung $\%$ C3\%84rztliche_Psychotherapie.pdf (Zugriff 14.08.2014)

Hand I: Verhaltenstherapie - Quo vadis seit dem PTG? Verhaltenstherapie 2001;11:284-286.
Hand I: Verhaltenstherapie - Schutz vor Mythologie und Magie in der Psychotherapie? Verhaltenstherapie 2003;13:161-163.

Hand I: Generationswechsel und Weiterentwicklung von Psychotherapie - Fakt oder Fiktion? Vortrag 37 (DVD). Langeooger Psychotherapiewoche, 16.-21.6.2008. Müllheim-Baden, Auditorium-Netzwerk, 2006.

Hand I: Strategisch-systemische Aspekte der Verhaltenstherapie. Wien, Springer, 2008

Hand I: Exposition und Konfrontation; in Linden M, Hautzinger M (eds): Verhaltenstherapiemanual, ed 11. Heidelberg, Spinger, 2011, pp 139-148.

Hand I: Expositionstraining (Konfrontationstraining); in Senf W, Broda M, Wilms B (eds): Techniken der Psychotherapie. Stuttgart, Thieme, 2013, pp 164171.

Hand I, Kegat S: Geldflüsse und Aktivitäten seit dem ersten Glücksspielstaatsvertrag: Wie viel, wohin, wofür und mit welchem Ergebnis? Konturen 2013; 5:33-39.
Hand I, Schroeder-Hartwig K, Weiss A, Friedrich B: Verhaltens«Therapeuten» aus der Kranken«Pflege» - 30 Jahre Modellprojekt an der Verhaltenstherapie-Ambulanz des Universitätsklinikums Hamburg-Eppendorf. Verhaltenstherapie 2014;24: 193-200.

Hillebrand T, Niedermeier N: Intensive Expositionsbehandlung bei schweren Zwängen - zwei Modelle aus der Praxis. Verhaltenstherapie 2014:24:201-210

Kassenärztliche Bundesvereinigung (KBV): Qualitätsbericht, Ausgabe 2013. www.kbv.de/media/sp/ KBV_Qualitaetsbericht_2013.pdf (Zugriff 14.08. 2014).

Linden M: Verhaltenstherapie - Totgesagte leben länger. Verhaltenstherapie 2013;23:72-73.

Malinke S: Konzept der GKV-SV zur Weiterentwicklung der ambulanten Versorgung. Vortrag zur Gutachtertagung der KBV. Berlin, 2014.

Techniker Krankenkasse (TK): Thesen zur ambulanten Psychotherapie, 2013.www.tk.de/tk/020-positionen/tk-psychotherapie/515658 (Zugriff 14.08.2014).

Verband der Ersatzkassen (VdEK): Ambulante Psychotherapie: Versorgung verbessern, 2013. $w w w$. vdek.com/magazin/ausgaben/2013-0708/fokusambulante-psychotherapie.html (Zugriff 14.08.2014). 